\title{
ANAESTHESIA PRACTICES FOR CAESAREAN SECTION AMONGST NON- PHYSICIAN ANAESTHESIA PROVIDERS AT AN ETHIOPIAN REFERRAL HOSPITAL
}

Victoria Emma White ${ }^{1}$, Gebrehiwot Asfaw ${ }^{2}$, Jolene Nina Moore ${ }^{3}$

1. Royal Free Hospital, London, UK

2. Bahir Dar University, Bahir Dar, Ethiopia

3. University of Aberdeen, Aberdeen, UK

$\underline{\text { Key words: }}$ obstetric, anaesthesia, Caesarean

\begin{abstract}
$\underline{\text { Abstract }}$
Sub-Saharan Africa faces high rates of maternal mortality and there is an urgent need to reduce this. Shortfalls in access to safe surgery and anaesthetic care result in avoidable maternal death. Providing quality training to anaesthesia providers is of key importance to reduce mortality.
\end{abstract}

This mixed methods prospective study incorporated workplace observations of anaesthesia for Caesarean section (CS), a paper-based questionnaire and semi-structured, face-to-face interviews in Felege Hiwot Referral Hospital in Ethiopia.

A total of $67 \mathrm{CS}$ cases under spinal anaesthesia provided by 12 non-physician anaesthetists were observed and a $92 \%(n=11)$ response rate to questionnaires obtained. Deficiencies were observed in communication, pre-operative assessment, spinal height evaluation and application of lateral tilt, while interviews revealed anaesthesia provider perceptions of hierarchy within the surgical team and deficiency in anticipation of potential complications.

This study suggests focusing on communication and anticipation of complications could aid providers in preventing and preparing for complications.

\section{$\underline{\text { Introduction }}$}

Reducing maternal mortality is a global priority. Demonstrating the greatest single health disparity between high and low income countries, over $94 \%$ of maternal deaths occur in low and middle income countries (LMIC), and the majority are preventable. ${ }^{1}$ Ethiopia has previously been identified as one of six countries contributing towards more than half of global maternal deaths, and despite improvements, the maternal mortality ratio (MMR) remains high. ${ }^{2-4}$ Around $75 \%$ of maternal deaths are associated with complications that require surgery and anaesthesia. ${ }^{4}$ The African Surgical Outcomes Study (ASOS) demonstrated that maternal mortality following CS is fifty times higher in sub-Saharan Africa than in high-income countries. ${ }^{5}$ The Lancet Commission on Global Surgery has highlighted the current shortfalls in provision of access to safe surgery, and a target date of 2030 has been set to achieve the goal of universal safe, accessible and affordable surgical and anaesthesia care when needed. ${ }^{6}$ 
Anaesthesia contributes disproportionately to maternal mortality in sub-Saharan Africa. Risk factors include inadequate training of anaesthesia providers, shortages of skilled providers, limited resources, and complications in the perioperative period. ${ }^{7-10}$ Access to training and professional development in anaesthesia has become a focus for improving maternal care and, ultimately, the outcome. ${ }^{11,12}$

The anaesthesia workforce in sub-Saharan Africa is considerably under-resourced. In Ethiopia, one of the world's most populous countries with over 114 million inhabitants. ${ }^{13}$ Figures from the 2016 World Federation of Societies of Anaesthesiologists (WFSA) workforce survey report only 51 physician anaesthesia providers (PAP); a PAP ratio of 0.05 per 100,000 population. ${ }^{14}$ An unbalanced distribution of healthcare providers is a recognised global problem. ${ }^{15}$ The majority of PAPs are concentrated around the capital, Addis Ababa, resulting in the use of non-physician anaesthesia providers (NPAPs) in a large part of the country. NPAPs make up a large proportion of the anaesthesia workforce in many African countries, with 22 distinct training pathways for NPAPs identified across 51African countries. ${ }^{16}$

In Ethiopia, a variety of training routes exist for NPAPs, from nurse diploma, to Bachelor of Science degree, to Masters of Science. Such a heterogeneous anaesthesia workforce contributes to variations in practice. In order to focus on the design of educational initiatives, it is important to understand current practices.

This study aimed to explore routine obstetric anaesthesia practice for CS by NPAPs in Felege Hiwot Referral Hospital (FHRH), a major referral centre in the Amhara region of Ethiopia serving a population of over 7 million people. FHRH conducts approximately 6000 deliveries per year with a CS rate of 35-40\% (2100-2400 CS per annum). The facility MMR is between 300-400 per $100,000 .^{1}$

\section{Methods}

This mixed methods study utilised direct workplace observations, a behavioural questionnaire and semi-structured face to-face interviews to assess the routine practices of NPAPs in CS. Ethical approval was obtained from the Institutional Review Board at Bahir Dar University, Ethiopia and informed consent was obtained from participating providers.

Over a 6-week period in January and February 2019, NPAPs were observed whilst providing anaesthesia for CS in FHRH. There were no PAPs working at FHRH, and NPAPs of all types of qualification working at FHRH were included. Observations were conducted during weekday daytime hours (08:00-16:00, Monday-Friday) and included both elective and emergency cases. Only cases undergoing spinal anaesthesia were included, as this is the accepted standard practice for anaesthesia for CS.

${ }^{1}$ FHRH obstetric department, personal communication, January 2019 
A standardised checklist was created containing demographics and 14 items of practice. Demographic data collected included the urgency of CS and length of time from clinical decision to induction of anaesthesia. Components of the checklist tool included communication, preoperative assessment and preparation, conduct of the procedure and infection prevention practices. These practice-related items closely matched those in the behavioural questionnaire. There was also space to record additional free text information pertaining to practice.

The behavioural questionnaire, created by The Change Exchange, a UK-based implementation science collaboration, has been utilised previously by the WFSA for monitoring and evaluation purposes during educational interventions (unpublished). This questionnaire features key tasks that would be expected to be performed routinely for spinal anaesthesia for CS. The questionnaire included questions relating to resource availability in participants' workplace. The questionnaire asked participants to answer using either a 0-10-point scale or a 'yes', 'no', 'not sure' structure, depending on the nature of the individual question. Questions with the 0-10-point scale were structured to ask in how many out of 10 cases they would usually perform X [the specific task]. This 10-point scale was converted into 'never', 'rarely', 'sometimes', 'usually' and 'always' by grouping the numerical answers of 0 as 'never', 1-3 as 'rarely', 4-6 as 'sometimes', 7-9 as 'usually', and 10 as 'always'.

Following the six-week observation period, the same cohort of NPAPs were asked to complete the behavioural questionnaire. These were distributed following the observation period to prevent any impact of the content of the questionnaire on workplace practice. Questionnaires were in English language; medical and healthcare-related training in Ethiopia is usually delivered in both Amharic and English language, so all providers had a reasonable level of English language capability.

Face-to-face interviews were conducted by VEW with a small number of NPAPs; these were of a semi-structured format. Interview questions were designed to explore practices where there was either a discrepancy between the observed practice during workplace observations and the selfreported practice in questionnaire answers, or where an area of practice was rarely or never performed. Interviews were conducted in the English language, recorded and later transcribed.

\section{$\underline{\text { Results }}$}

Sixty-seven CS cases were observed during daytime hours within this period, of which $62.7 \%$ $(n=42)$ were emergencies. Twelve NPAPs were observed, representing $86 \%(n=14)$ of those working at the institution during the study. These included seven-degree level (BSc) anaesthetists and five Level V (diploma nurse) anaesthetists. The number of years in anaesthetic practice had a median (IQR [range]) of 4.3 (1.5-5.8[1-10]). The median number of cases in which each NPAP was observed was $8.25(2-10.25[1-13])$. 
The most common indications for CS were: previous CS 30 (44.7\%); foetal distress 13 (19.4\%); and failure to progress/obstructed labour $12(17.9 \%)$. Other indications included failed induction of labour, breech presentation, foetal macrosomia, pre-eclampsia, twin pregnancy, retroviral infection, vaginal fistula and suspected placental abruption. Several cases had more than one indication. A preoperative assessment prior to anaesthesia was conducted in 22 (32.8\%) of cases, despite prior knowledge of elective lists and a median time from decision to induction of anaesthesia for emergency cases of one hour (0.5-1.5 [0.17-3.5]). NPAP and obstetrician discussed case details prior to surgery for emergencies cases only.

The World Health Organization (WHO) Surgical Safety Checklist (SSC) was present in all cases, although its utilisation was incomplete. The first (before induction of anaesthesia) and last (before patient leaves the operating room) steps were always performed; however, omission of the surgical pause completed before skin incision occurred in all cases. Although preparation of drugs for induction of general anaesthesia, should it be required, was not observed in any case, induction agents were noted by the observer to be available in the vicinity.

Sterility during spinal anaesthesia, provision of antibiotics and regular monitoring of patient's vital signs (heart rate, blood pressure, oxygen saturations) was observed in all cases. Measurement of height of the spinal block was not performed in any case. A left lateral tilt to reduce aortocaval compression was applied in $37(55.2 \%)$. Further details of individual observed behaviors are illustrated in Figure 1.

There was a $91.7 \%$ (11) questionnaire response rate, the results of which are summarised in Figure 2. All respondents reported having the necessary equipment and drugs for spinal anaesthesia in the period preceding the completion of the questionnaire. No respondent reported never performing any aspect of practice. Those practices which had reports of being rarely performed include communication with obstetrician, ensuring a vasopressor is available, utilising the WHO SSC and giving intravenous antibiotics. Use of the WHO SSC and provision of antibiotics were considered the role of the scrub nurse and midwife.

Four anaesthesia providers agreed to participate in face-to-face, semi-structured interviews. Participants $(\mathrm{P})$ described a lack of professional recognition towards anaesthesia providers by surgeons and hierarchical systems that impacted on their ability to perform certain elements of anaesthesia practice, or on their behaviours. This was apparent when they discussed communication with obstetricians, where participants described the obstetrician determining which cases or details would be discussed, and the main determinant of this being complexity of the case: "sometimes - critical patients, or complex electives" [P1], as well as a lack of inclusion in decision making: "they do not make you part of the decision" [P4] and omission of components of the WHO SSC: "the residents want to rush when there is an emergency, so they said no" [P4]. Interview participants understood the reason for ascertaining height of the spinal block yet felt cardiovascular stability and sufficient motor impairment negated the need for complete assessment: "we check on their vital signs... if we notice hypotension then we will check" [P1]; "if their vital signs are ok, autonomically it is ok" [P3]; "if they can't lift their legs it is ok" [P2]. 


\section{Discussion}

This study reports on anaesthesia practices for CS by NPAPs at an Ethiopian referral hospital. High standards of practice were observed in patient monitoring and infection prevention practices. Deficiencies were observed in pre-operative assessment communication, WHO SSC completion, measuring spinal block height and application of left lateral tilt. Self-reported practice and observed practice were similar in highlighting these deficiencies, with the exception of preoperative assessment, where self-reporting did not identify this deficiency; application of left tilt was not compared.

Pre-operative assessment was conducted in only one third of cases. Although induction agents were not prepared in case of emergency, they were always available in the vicinity. Despite always discussing case urgency, case details and management was only discussed in emergencies with selective communication driven by obstetricians. The WHO SSC can create an atmosphere for effective communication. ${ }^{17}$ Despite its utilisation, the pause point prior to skin incision was omitted. An earlier study at FHRH reported similar findings, with WHO SSC use in all obstetric surgical cases following phased implementation, and the surgical pause point the least completed component. $^{18}$

Spinal hypotension can lead to maternal and foetal compromise. Placement of a left lateral tilt to minimise aortocaval compression should be applied and vasopressors should be available ${ }^{19}$; left lateral tilt was applied in $55.2 \%$ and vasopressors were available in $80 \%$ of cases. Assessment of height of spinal block was not performed in any case. Providers reported only doing so when the patient became unstable suggestive of a high spinal, indicating reactive versus anticipatory behavior.

There is limited literature reporting anaesthesia practice for CS amongst NPAPs; a recent study in Kenya observed clinical behaviour of NPAPs following obstetric anaesthesia training to determine its impact. ${ }^{20}$ Practice not adhered to according to training recommendations included performing the WHO SSC, checking spinal block height and patient monitoring. Behaviour observed in line with training included pre-operative preparation, sterile technique for spinal anaesthesia, teamwork and communication.

This study was limited by the number of providers and frequency with which each individual was observed resulting in a small sample size. There was a range in number of observations per provider which may result in bias towards the behaviour of the individual observed more frequently. Questionnaire and interview responses may have been impacted by the understanding of English or by the concern for consequences, while interviews included a small number of providers, thus limiting conclusions drawn. The study was conducted at one facility and could be impacted by other factors present at this facility; therefore, a larger scale study encompassing a broader assessment of practice and more than one facility is recommended. 
This study provides insight into the practices of NPAPs during anaesthesia for CS at an Ethiopian Referral Hospital and highlighted deficiencies in specific areas of practice; in particular, those related to communication and elements of obstetric anaesthesia specific practice. Identifying these practice deficiencies may aid direction of future educational initiatives in this region.

\section{Acknowledgments}

The authors wish to thank the WFSA for providing permission to utilise the behavioural survey.

\section{Declarations of Interest}

The authors declare that there is no conflict of interest.

\section{Funding}

This project received funding in the form of a travel bursary from the Association of Anaesthetists, the Obstetric Anaesthetists Association, and the Royal College of Medicine, to enable the conduct of this project by the primary author. 


\section{$\underline{\text { References }}$}

1. Trends in maternal mortality: 2000 to 2017: estimates by WHO, UCICEF, UNFPA, World Bank Group and the United Nations Population Division. Geneva: World Health Organization; 2019.

2. Alkema L, Chou D, Hogan D, et al. Global, regional, and national levels and trends in maternal mortality between 1990 and 2015, with scenario-based projections to 2030: a systematic analysis by the UN Maternal Mortality Estimation Inter-Agency Group. Lancet. 2016; 387 (10017): 462-74.

3. Hogan MC, Foreman KJ, Naghavi M, et al. Maternal mortality for 181 countries, 1980-2008: a systematic analysis of progress towards Millennium Development Goal 5. The Institute of Health Metrics and Evaluation (IHME) at the University of Washington in Seattle, USA. Lancet. 2010; 375:1609-1623.

4. Say L, Chou D, Gemmill A, et al. Global causes of maternal death: a WHO systematic analysis. Lancet. 2014;2(6):PE323-E33

5. Biccard BM, Madiba TE, Kluyts H-L, et al. Perioperative patient outcomes in the African Surgical Outcomes Study: a 7-day prospective observational cohort study. Lancet. 2018;391:1589-98

6. Meara JG, Leather AJM, Hagander L, et al. Global Surgery 2030: evidence and solutions for achieving health, welfare and economic development. Lancet 2015; 386(9993):569-624

7. Ouro-Bang'na Maman AF, Tomta K, Ahouangbévi S, et al. Deaths associated with anaesthesia in Togo, West Africa. Tropical Doctor. 2005;35:220-222

8. Hansen D, Gausi SC, Merikebu M. Anesthesia in Malawi: complications and deaths. Tropical Doctor. 2000;30:146-149

9. Glenshaw M, Madzimbamuto FD. Anaesthesia associated mortality in a district hospital in Zimbabwe: 1994 to 2001. Central African Journal Medicine. 2005;51:39-44

10. Sobhy S, Zamora J, Dharmarajah K, et al. Anaesthesia-related maternal mortality in lowincome and middle-income countries: a systematic review and meta-analysis. Lancet Glob Health. 2016;4:e320-27

11. Hodges SC, Mijumbi C, Okello M, et al. Anaesthesia services in developing countries: defining the problems. Anaesthesia. 2007;62:4-11

12. Enright A, Grady K, F E. A New Approach to Teaching Obstetric Anaesthesia in LowResource Areas. Women's Health. 2015;37(10):880-4

13. Review WP. Ethiopia Population. Available from: https://worldpopulationreview.com/countries/ethiopia-population/ [Accessed 13/06/20]

14. Kempthorne P, Morriss W, Mellin-Olsen J, et al. The WFSA Global Anesthesia Workforce Survey. Anaesthesia and Analgesia. 2017;125(3):981-90

15. Dussault G, Franceschini MC. Not enough there, too many here: understanding geographical imbalances in the distribution of the health workforce. Human Resource Health 2006;4:12

16. Law T, Bulamba F, Ochieng JP et al. Anaesthesia Provider Training and Practice Models: A Survey of Africa. Anaesthesia and Analgesia 2019; 129(3); 839-8446 
17. Weiser TG, Haynes A. Ten years of the Surgical Safety Checklist. British Journal of Surgery. 2018;105:927-

18. Ellis E, Nor AIM, Pimentil I, et al. Improving Surgical and Anaesthesia Practice: Review of the use of the WHO Surgical Safety Checklist in Felege Hiwot Referral Hospital, Ethiopia. BMJ Open Quality 2017; 6:u207104.w6251 doi:10.1136/bmjquality.u207104.w6251

19. K Kinsella SM, Carvalho B, Dyer RA, et al. International concensus statement on the management of hypotension with vasopressors during caesarean section. Anaesthesia 2018;73(1):71-92

20. Lilaonitkul M, Mishra S, Pritchard N, Andhoga J, Olang P, Kibet EB, et al. Mixed method analysis of factors influencing change in clinical behaviours of non-physician anaesthetists in Kenya following obstetric anaesthesia training. Anaesthesia 2020; Advance online publication. doi:10.1111/anae.15091

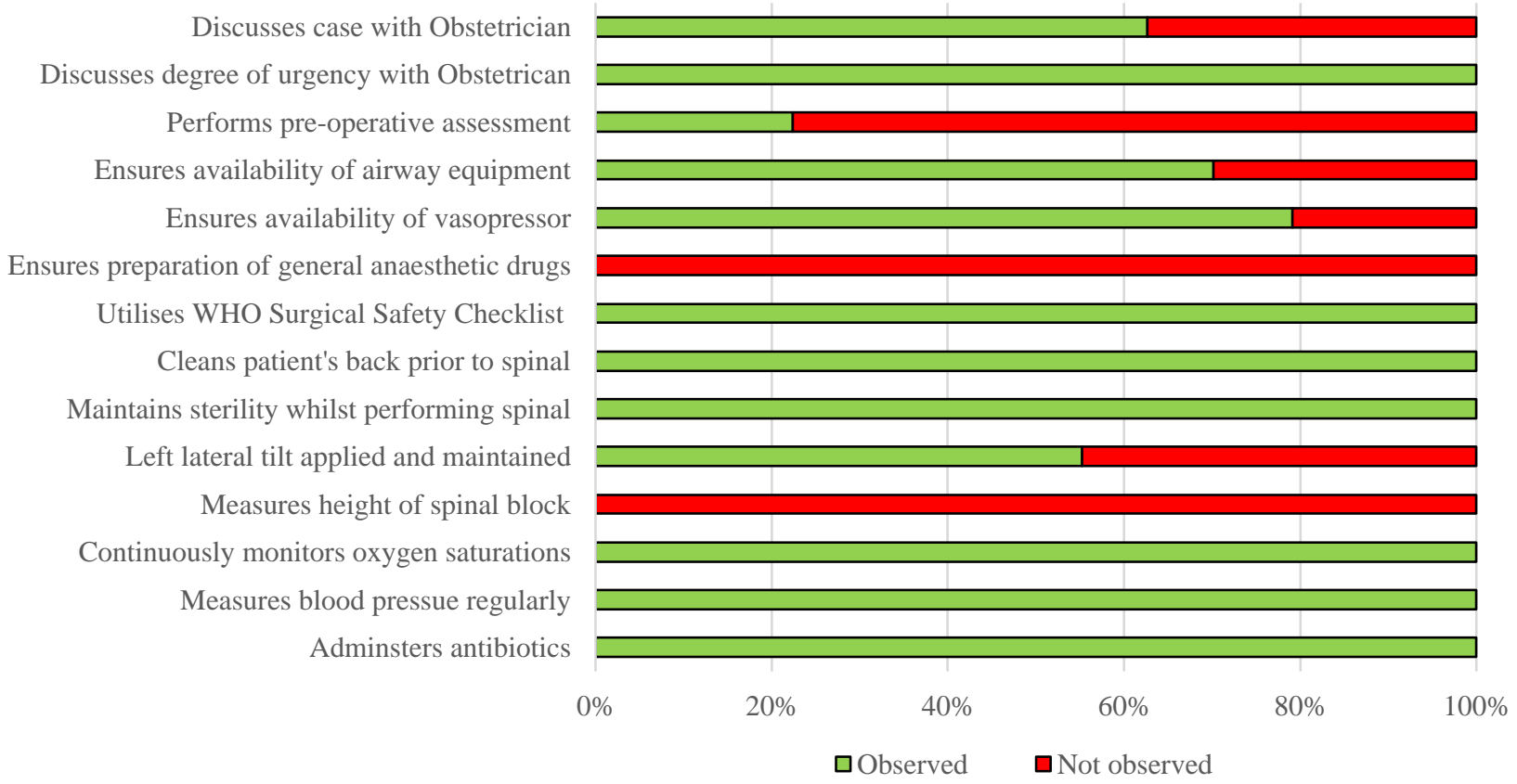

Figure 1. Observed practices of NPAPs during anaesthesia for CS 
Discusses case with obstetrican

In pre-eclampsia, discusses case with obstetrician

Discusses degree of urgency with obstetrican

Performs a pre-operative assessment

Ensures availabilty of airway equipment

Ensures availabilty of vasopressor

Ensures preparation of general anaesthetic drugs

Utilises WHO Surgical Safety Checklist

Cleans patient's back prior to spinal

Measures height of spinal block

Regularly monitors blood pressure

Adminsters antibiotics

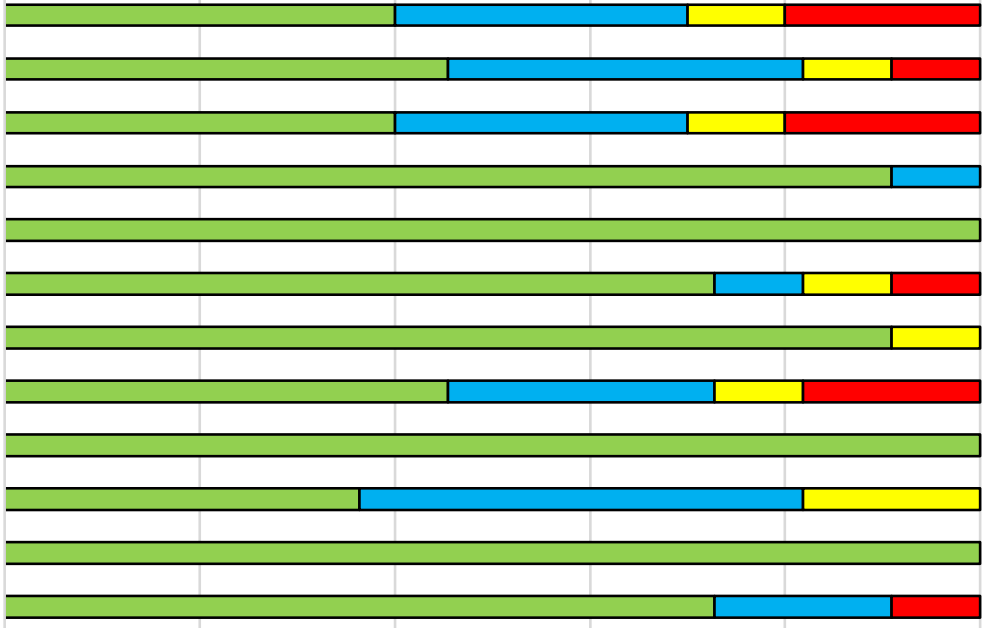

$0 \%$

$20 \%$

$40 \%$

$60 \%$

$80 \%$

$100 \%$

$\square$ Always

口Usually

$\square$ Sometimes

口Rarely

Figure 2. Self-reported practices of NPAPs during anaesthesia for CS 